\title{
Erratum to Chapter: Pain-Modulating Peptides in Spider Venoms: Good and Evil
}

\section{Sylvie Diochot}

Owing to an error on the part of Springer the first name and the family name of the author were transposed in the initially published version of this chapter.

The correct name is: Sylvie Diochot

The online version of the updated chapter can be found at http://dx.doi.org/10.1007/978-94-0076389-0_18

\section{S. Diochot}

Institut de Pharmacologie Moléculaire et Cellulaire, CNRS UMR7275, Université de Nice-Sophia Antipolis, Valbonne, France

e-mail: diochot@ipmc.cnrs.fr 